\title{
A PRICING MODEL IN A SENSITIVE INSURANCE MARKET
}

\author{
By FRANCO MORICONI \\ Institute of Mathematics, University of Rome
}

\section{INTRODUCTION}

A great attention has been devoted, in the actuarial literature, to premium calculation principles and it has been often emphasized that these principles should not only be defined in strictly actuarial terms, but should also take into account the market conditions (Bühlmann (1980), de Jong (1981)).

In this paper we propose a decision model to define the pricing policy of an insurance company that operates in a market which is stratified in $k$ risk classes $\mathscr{C}_{j}$.

It is assumed that any class constitutes a homogeneous collective containing $\mathcal{N}_{j}$ independent risks $S_{j}(t)$ of compound Poisson type, with the same intensity $\lambda_{j}$. The number $n_{j}$ of risks of $\mathscr{C}_{j}$ that are held in the insurance portfolio depends on the premium charged to the class by means of a demand function which captures the concept of risk aversion and represents the fraction of individuals of $\mathscr{C}_{j}$ that insure themselves at the annual premium $x_{j}$.

With these assumptions, the return $Y$ on the portfolio is a function of the vector $\boldsymbol{x}=\left(x_{1}, x_{2}, \ldots, x_{k}\right)$ of the prices charged to the single classes (and of the time) and $\boldsymbol{x}$ is therefore the decision policy instrument adopted by the company for the selection of the portfolio, whose optimal composition is evaluated according to a risk-return type performance criterion.

As a measure of risk we adopt the ultimate ruin probability $q(w)$ that, in the assumptions of our model, can be related to a safety index $\tau$, by means of Lundberg-de Finetti inequality. Even though it has been widely debated in the actuarial field, the use of $q(w)$ offers undeniable operational advantages. In our case the safety index $\tau$ can be expressed as a function of $\boldsymbol{x}$ and therefore, in the phase of selecting an efficient portfolio, it becomes the function to be maximized, for a given level $M$ of the expected return.

For $\tau$, a quadratic approximation can be given that seems to be acceptable as long as the aggregate loading is not "too high". An assumption that does not exclude, among other things, the possibility of heavy loadings in a number not too large of individual cases.

Once the form of the efficient frontier has been determined, the final step of the decision problem of the company is to select the portfolio that maximizes a utility function of the form $u(M, V)$, that is the portfolio represented as the tangency point between the efficient frontier and the "highest possible" indifference curve. It could be pointed out that, in the model, the validity of variance as a risk measure of the portfolio does not depend on the possibility of achieving an acceptable quadratic approximation of the utility function, but on the goodness of the approximation obtained for the ruin probability, that we have chosen as a stability criterion for the company. 
It is to be noted that, in our assumptions, we can achieve a stratification more refined than the one obtained solely based on the characteristics of the risk process $S(t)$, since we can consider classes that differ only for the risk aversion. This can be related to the introduction of a multivariate measure of risk aversion inside the collective, as suggested, e.g., by Sengupta (1981).

On the other hand the form and the rationale of the results continue to be valid in all the cases in which we can obtain an expression that relates $q(w)$ to the decision variables (i.e., the prices) and the endogenous quantities of the risk process. This is the case, for example, in the martingale assumptions on $Y(t)$, as discussed by de Finetti (1939) and developed by Gerber (1981) in the study of an autoregressive model.

\section{THE MODEL}

\subsection{Preliminaries}

Let us consider the risk process $\{S(t) ; t \geqslant 0\}$, that represents the sum of claim amounts incurred in $[0, t)$ in a given insurance portfolio. The accumulated claims up to time $t$ can be represented as a random sum

$$
S(t)=\sum_{r=1}^{N(t)} X_{r},
$$

with d.f. $F_{S}(x, t)=P\{S(t) \leqslant x\}$. The process $\{N(t) ; t \geqslant 0\}$, with distribution $p_{n}(t)$, $(n=0,1, \ldots)$, counts the number of claims in $[0, t)$ and the set of r.v. $\left\{X_{r} ; r=\right.$ $1,2, \ldots\}$ represents the amount of the $r$ th claim incurred in $[0, t)$. We can suppose that the m.g.f. $\chi_{r}(u)=E\left\{e^{u X_{r}}\right\}$ is finite for some $u \neq 0$.

We shall assume that the collective premium function of the risk (sum of premiums earned in the time interval $[0, t)$ ) is non-random and we shall denote it by $\pi(t)=E\{S(t)\}+l(t)$, that is as a sum of the (aggregate) net premium $E\{S(t)\}$ and the (aggregate) risk loading $l(t)$. As generally accepted in the actuarial literature, we shall assume $l(t) \geqslant 0$, since we shall disregard investment income in premium calculation. In fact, as shown by Kahane (1979), negative loadings could be justified by considerations on the cost of the capital and on the rates of investment. Meaningful loading formulas are obtained for instance by choosing $l(t)$ to be proportional to the expected value (supposed as positive) or to the variance of $S(t)$, that is

$$
l(t)=\eta E\{\boldsymbol{S}(t)\}, \quad \eta \geqslant 0,
$$

or

$$
l(t)=\beta \operatorname{Var}\{S(t)\}, \quad \beta \geqslant 0 .
$$

Besides the investment income, we shall neglect also the administrative costs and we shall indicate by $Y(t)=\pi(t)-S(t)$ the return on the insurance portfolio up to time $t$. Then the liquidity of the company can be represented by the risk 
reserve $R(t)=w+Y(t)$, being $w=R(0)$ the initial free capital; namely we have

$$
R(t)=w+E\{S(t)\}+l(t)-\sum_{r=1}^{N(t)} X_{r}
$$

One of the most natural assumptions on the process $S(t)$ is that the r.v. $X_{r}$ are independent with common d.f. $F_{X}(x)$ independent on time and such that $F_{X}(0)=0$ (positive risk sums). If we suppose, following $\mathrm{F}$. Lundberg, that $N(t)$ is a Poisson process with intensity $\lambda$, the risk process $S(t)$ becomes a compound Poisson process, with m.g.f.

$$
\varphi_{S}(u, t)=\exp \{\lambda t[\chi(u)-1]\},
$$

where $\chi(u)$ is the common m.g.f. of the $X_{r}$, and with expected value $E\{S(t)\}=$ $\lambda E\{X\} t$. Furthermore the risk loading becomes a linear function of time, i.e., $l(t)=l \cdot t$, whether one uses the expected value principle or the variance principle.

\subsection{The Risk Classes}

The foregoing classical model can be used to describe the riskiness of the portfolio of a given insurance line. Let us now suppose that the insurance market relative to this line is stratified in $k$ risk classes $\mathscr{C}_{j},(j=1,2, \ldots, k)$ according to the following hypotheses

(a) $\mathcal{N}$ stochastically independent individual risks are in the market.

(b) The class $\mathscr{C}_{j}$ is a homogeneous collective consisting of $\mathcal{N}_{j}$ (being $\sum_{j=1}^{k} \mathcal{N}_{j}=$ $\mathcal{N})$ risks $S_{j}(t)$ which are compound Poisson processes with the same intensity $\lambda_{j}$. The classes are assumed to be ordered in such a way that $\lambda_{1} \leqslant \lambda_{2} \leqslant \cdots \leqslant \lambda_{k}$.

(c) The m.g.f. $\chi(u)$ is the same for all the classes.

(d) For any individual risk in the class $\mathscr{C}_{j}$ the premium $x_{j} t=\left(\lambda_{j} E\{X\}+l_{j}\right) t$ is charged. Therefore $x_{j}$ and $l_{j}$ represent the annual premium and the annual loading relative to these risks, respectively.

Denoting then by $n_{j}$ the number of risks of the class $\mathscr{C}_{j}$ that are held in the portfolio, for the property of infinite divisibility one has $\lambda=\sum_{j=1}^{k} \lambda_{j} n_{j}$ and the return on the whole portfolio

$$
Y(t)=\left(\sum_{j=1}^{k} x_{j} n_{j}\right) t-\sum_{j=1}^{k} \sum_{\mathscr{Q}_{i}} S_{j}(t)
$$

has m.g.f.

$$
\varphi_{Y}(u, t)=\exp \left\{\left(\sum_{j=1}^{k} x_{j} n_{i}\right) u t+[\chi(-u)-1]\left(\sum_{j=1}^{k} \lambda_{j} n_{j}\right) t\right\}
$$

\subsection{Anti-Selection}

In this situation, if a company $A$ decided to collect an aggregate annual premium $\pi(1)=\pi$ to protect itself against unfavourable outcome of the risk process, it 
could be led to charge to each contractholder the "average" premium $\pi / n$, with $n=\sum_{i=1}^{k} n_{j}$. In fact such a choice would offer the advantage of minimizing the administrative costs. But if it were possible to know the risk class to which the contracts (not necessarily all of them) belong, it would be easy for a competing firm $B$ to collect policies concerning low-risk individuals, by charging them a premium less then $\pi / n$. On the other hand, the individuals that are more exposed to risk would be spurred to insure themselves with the company $A$, considering as advantageous the average premium $\pi / n$. The effect of such an anti-selection mechanism would then be an alteration of the company $A$ 's portfolio composition, such that it would increase even considerably the probability of a negative evolution of the process $Y(t)$. So the choice of the value $\pi$ would turn out to be inadequate.

Therefore if we make the necessarily schematic and simplifying assumption that the company and the policyholders are in a state of perfect information on the parameters of the risk process and in particular on the value of the intensity $\lambda_{j}$, the choice of the premiums will have a significant influence on the composition of the portfolio.

REMARK. The assumption of perfect information finds a different formulation within the subjectivistic theory of probability. In fact in this context it means that the parts are in agreement on the values of the probabilities. The problem was discussed, e.g., by Pressacco (1979), who questioned whether a subjective fair price can be given an objective meaning.

The possibility of different probability evaluations has been considered, e.g., by de Ferra (1968) and Volpe di Prignano (1974). In these cases the "advantageousness" of an insurance contract can be studied by defining an indifference premium that differs from the net premium both in consideration of the risk aversion and because of the diverse evaluations of the probabilities. The importance of these problems has also been emphasized by Rothschild and Stiglitz (1976), who studied the equilibrium in a competitive insurance market in a state of imperfect information.

In any case, the dependence of premium determination upon the market conditions is the basic assumption in the economic models of insurance market proposed, e.g., by Bühlmann (1980) and de Jong (1981).

\subsection{Demand Function and Risk Aversion}

We are thus led to introduce in the model a dependence of $n_{j}$ on the premium charged to the class $\mathscr{C}_{j}$, i.e., $n_{j}=n_{j}\left(x_{j}\right),(j=1,2, \ldots, k)$. Following Cacciafesta (1970), we shall make the rather natural assumption

$$
n_{j}(x)=\mathcal{N}_{j} d_{j}(x), \quad(j=1,2, \ldots, k),
$$

where the demand function $d_{j}(x)$ (that we, for sake of simplicity, shall treat as a real-valued function) represents the fraction of individuals of the class $\mathscr{C}_{j}$ that insure themselves at the annual premium $x$ and therefore it expresses the 
sensitivity of $\mathscr{C}_{j}$ to the price that is charged. If one were to represent this function (for given $j$ ) as a set of random variables $\left\{d_{j}(x) ; x \geqslant 0\right\}$, with probability distributions that are chosen based on statistical observations, besides procedural and estimative complications, the highly significant relation between demand function and risk aversion would be mistreated.

Instead of strictly describing the function $d_{i}(x)$, it therefore appears more significant to refer to a deterministic model characterized by

$$
d_{j}(x)= \begin{cases}1, & \text { for } 0 \leqslant x<\lambda_{j} E\{X\}, \\ \frac{x_{i}^{*}-x}{l_{j}^{*}} ; & \text { for } \lambda_{j} E\{X\} \leqslant x \leqslant x_{i}^{*}, \\ 0, & \text { for } x>x_{j}^{*},\end{cases}
$$

with $x_{j}^{*}=\lambda_{j} E\{X\}+l_{j}^{*}, l_{j}^{*} \geqslant 0,(j=1,2, \ldots, k)$. Evidently $x_{j}^{*}$ can be seen as a measure of the risk aversion of the class $\mathscr{C}_{j}$ as a whole. It is interesting to note that the function $d_{j}(x)$ can be interpreted as the probability that an individual of $\mathscr{C}_{j}$ chosen at random insures himself, provided that the decisions of the individuals are stochastically independent; in this case $n_{j}(x)$ is to be understood as the expected number.

If we accept the assumption that the risk to which an individual is exposed is small relative to his wealth $c$, i.e., $E\{S(1)\} \ll c$, if his utility function $u(z)$ can be expanded in a Taylor series around $c$ and if we limit ourselves to a second-order approximation, then we obtain a quadratic utility function

$$
u(c+z)=z-\frac{1}{2} r(c) z^{2},
$$

where $r(c)=-u^{\prime \prime}(c) / u^{\prime}(c)$ is the Arrow-Pratt (local) risk aversion, or, in other terms, the (local) propension to insurance (in the actuarial applications $r$ is generally supposed as a decreasing function of $c$ ). If all the individuals of the class $\mathscr{C}_{j}$ have the same value $r_{j}$ of risk aversion, then $x_{j}^{*}$ and $l_{j}^{*}$ represent respectively the maximum acceptable premium and the maximum acceptable loading by each one of them.

Because of the Poisson assumptions on the risk process, one can prove that

$$
l_{j}^{*} \simeq \frac{1}{2} r_{j} \lambda_{j} E\left\{X^{2}\right\}
$$

by expressing $l_{j}^{*}$ according to the variance principle, i.e., $l_{j}^{*}=\beta_{j}^{*} \operatorname{Var}\left\{S_{j}(1)\right\}$, relation (2.3) gives: $\beta_{j}^{*} \simeq \frac{1}{2} r_{j}$.

The foregoing considerations suggest, among other things, that it includes in the model the possibility of a stratification more refined than the one obtained solely based on the characteristics of the risk process $S(t)$, since one can take in consideration classes that differ only for the risk aversion (without contradicting the hypotheses made in (2.2)).

The introduction of the functions $n_{j}(x)$ brings about that all the variables endogenous to the risk come to depend upon the choice of the vector $x$ of the prices charged to the classes. From relation (2.1) one can, for example, derive 
the expression of the expected return on the portfolio

$$
M(x, t)=E\{Y(x, t)\}=\left[\sum_{j=1}^{k} N_{j} l_{j} d_{j}\left(x_{j}\right)\right] t
$$

and that of the variance of the portfolio return

$$
V(\boldsymbol{x}, t)=\operatorname{Var}\{Y(\boldsymbol{x}, t)\}=\left[\sum_{j=1}^{k} \mathcal{N}_{j} \lambda_{j} d_{j}\left(x_{j}\right)\right] E\left\{X^{2}\right\} t
$$

\subsection{The Probability of Ruin}

Because of the form of the demand curves that we have assumed, the charging of a premium $x_{j}>x_{j}^{*}$ is entirely equivalent to a refusal by the company of the risks belonging to the class $\mathscr{C}_{j}$; the choice of the price vector thus seems to be a significant and reliable means for the portfolio selection.

The process $Y(x, t)$ can be evaluated in terms of risk-return, that is by defining a performance criterion explicitly in terms of expected return and of portfolio risk and by choosing the best composition according to this criterion.

Many and plausible measures of risk can be proposed and adopted, but in our case it is natural to consider the probability of ruin before time $t, q(w, t)$, which moreover is the most investigated stability criterion in the actuarial literature and is also widely adopted in the administrative policy of the insurance companies. As can be seen, for example, in Seal (1979), it is generally rather complicated to evaluate $q(w, t)$ and this is also the case in models based on Poisson assumptions. It is instead rather easy to obtain useful results in the asymptotic case, i.e., for $q(w)=\lim _{t \rightarrow \infty} q(w, t)$.

In fact, with the assumptions of our model, the following classical result holds

$$
q(w) \leqslant e^{-\tau w},
$$

$-\tau$ being the negative root of

$$
E\left\{e^{u Y(t)}\right\}=1
$$

The inequality (2.6) was derived by F. Lundberg (1909) and by de Finetti (1939) using different methods. $\tau$, known as safety index, is also called adjustment coefficient, e.g., by Gerber (1981), who proposed a martingale theoretic approach to the ruin problem.

Generally, the right-hand side of (2.6) does not represent the ruin probability but provides an upper bound for it. However we are dealing with an "efficient" bound, because relation $(2.6)$ becomes an equality when the graph of the realizations of the process $Y(t)$ can not jump the barrier $-w$, that is if at the time of ruin there remains no margin of insolvency (Dubourdieu (1952)). 
From equations (2.1) and (2.7), $-\tau$ is the negative root of

$$
\chi(-u)-1=-u \frac{\sum_{j=1}^{k} x_{j} n_{j}\left(x_{j}\right)}{\sum_{j=1}^{k} \lambda_{j} n_{j}\left(x_{j}\right)}
$$

Because of the independence of such an expression from the time variable it is therefore sufficient to refer to a single-period model, as it was reasonable to expect, due to the fact that $Y(t)$ is a process with independent increments. All the endogenous quantities characteristic to the model will then be single-period (annual) quantities.

It is interesting to derive a quadratic approximation by using the property $\chi(-u)=1-E\{X\}+u^{2} E\left\{X^{2}\right\} / 2+o\left(u^{2}\right)$. From (2.8) we then obtain

which provides

$$
u E\{X\}-\frac{u^{2}}{2} E\left\{X^{2}\right\} \simeq u \frac{\sum_{j=1}^{k} x_{j} n_{j}\left(x_{j}\right)}{\sum_{j=1}^{k} \lambda_{j} n_{j}\left(x_{j}\right)}
$$

$$
\tau \simeq 2 \frac{1}{E\left\{X^{2}\right\}} \frac{\sum_{j=1}^{k} \mathcal{N}_{j} l_{j} d_{j}\left(x_{j}\right)}{\sum_{j=1}^{k} \mathcal{N}_{j} \lambda_{j} d_{j}\left(x_{j}\right)}=2 \frac{M(\boldsymbol{x})}{V(\boldsymbol{x})}
$$

where we denote $M(x)=M(x, 1)$ and $V(x)=V(x, 1)$. It should be pointed out that since the approximation is valid near the origin, then the less the quantity $\sum_{j=1}^{k} x_{j} n_{j} / \sum_{j=1}^{k} \lambda_{j} n_{j}$ exceeds the value of the derivative of $\chi(u)$ at the point $u=0$, the better the approximation is. This means that the results which we shall obtain will be much better, the closer we get to the fairness condition in the whole portfolio.

REMARK. The evaluation of the stability of an insurance company with an infinite planning horizon can raise doubts of a conceptual nature and in fact, in the past, the suitability of using the ultimate ruin probability has been widely debated (for a review, see Ammeter, Depoid and de Finetti (1957, p. 59)). The question has not remained limited strictly to the actuarial setting; for example, Massé (1964) has made use of the index $q(w)$ to compare the two notions of complete strategy and incomplete strategy. More recently, Ammeter (1970) has applied the ultimate ruin probability criterion in the study of the solvency problem of the european life insurance companies.

The parameter $\tau$ has been used even lately by Amsler (1978), who introduced it in his "general equilibrium equation of a collective risk", obtaining from it the definition of a solvency index.

\section{SELECTING THE OPTIMAL INSURANCE PORTFOLIO}

\subsection{The Programming Problem}

In the foregoing model the main problem faced by the company is to choose the price vector $\boldsymbol{x}$ so as to constitute an efficient portfolio, which has the maximum safety index for a given level $M$ of the expected return. It has then to solve the 
following programming problem

$$
\left\{\begin{array}{l}
\max \tau(x) \\
M(x)=M \geqslant 0 \\
x_{j} \geqslant \lambda_{j}, \quad(j=1,2, \ldots, k),
\end{array}\right.
$$

where the lower bounds on the $x_{j}$ are due to the fact that we have excluded negative loadings.

Since the factor $1 / E\left\{X^{2}\right\}$ seems to be irrelevant in the optimization problem, one can put this quantity equal to 1 , as for example would be the case if $S(t)$ were an ordinary Poisson process (unit jumps, $\chi(u)=e^{u}$ ). For sake of simplicity we shall indeed confine ourselves to this case, from now on, by putting moreover $E\{X\}=1$. Obviously, with these limitations the expected value principle and the variance principle turn out to coincide and we shall write $l_{j}=\eta_{j} \lambda_{j}$. It can be noted that in this case $\eta_{j}$ directly represents the Arrow-Pratt risk aversion in so far as, within the limits of the quadratic approximation of the utility function, one has $\eta_{j} \simeq \frac{1}{2} r_{j}$.

Recalling equation (2.9), problem (3.1) is equivalent to

$$
\left\{\begin{array}{l}
\min V(\boldsymbol{x}) \\
M(\boldsymbol{x})=M \geqslant 0 \\
x_{j} \geqslant \lambda_{j}, \quad(j=1,2, \ldots, k) ;
\end{array}\right.
$$

we are thus led to a mean-variance model.

Obviously, it is sufficient to study the problem (3.2) within the interval $D$ of the Euclidean $k$-space $\mathbb{R}^{k}$ :

$$
D \equiv\left\{\boldsymbol{x} \mid \lambda_{j} \leqslant x_{j} \leqslant x_{j}^{*} ; j=1,2, \ldots, k\right\} .
$$

In fact all the intervals for which $x_{j}>x_{j}^{*}$ for one or more values of $j$, that correspond to the exclusion of some risk classes, are equivalent to the cases $x_{j}=x_{j}^{*}$ and therefore are represented by intervals on the boundary of $D$.

Hence the problem (3.2) takes on the following explicit formulation

$$
\left\{\begin{array}{l}
\min \sum_{j=1}^{k} \frac{\mathcal{N}_{j}}{\eta_{j}^{*}}\left[-x_{j}+\left(1+\eta_{j}^{*}\right) \lambda_{j}\right] \\
\sum_{j=1}^{k} \frac{\mathcal{N}_{i}}{\eta_{j}^{*}}\left[-\frac{1}{\lambda_{j}} x_{j}^{2}+\left(2+\eta_{j}^{*}\right) x_{j}-\left(1+\eta_{j}^{*}\right) \lambda_{j}\right]=M \\
\lambda_{j} \leqslant x_{j} \leqslant \lambda_{j}\left(1+\eta_{j}^{*}\right), \quad(j=1,2, \ldots, k) .
\end{array}\right.
$$

This programming problem differs from those typical to the mean-variance models that are used in the portfolio analysis in that the objective function is linear, whereas the constraint is a quadratic function which contains the linear terms but in which the mixed terms are missing. The latter characteristic depends on the hypothesis of independence among the risks. 


\subsection{Deriving the Efficient Prices}

The constraint equation represents, when $M$ varies in $\mathbb{R}^{+}$, a portion of elliptic paraboloid in $k+1$ dimensions, whose vertex has the following coordinates

$$
\left\{\begin{array}{l}
M^{*}=\frac{1}{4} \sum_{j=1}^{k} \mathcal{N}_{j} \lambda_{j} \eta_{j}^{*}, \\
x_{j}=\left(1+\frac{\eta_{j}^{*}}{2}\right) \lambda_{j}, \quad(j=1,2, \ldots, k) .
\end{array}\right.
$$

Therefore the maximum expected return $M^{*}$ is obtained by choosing $x$ coinciding with the center $C$ of $D$, whose coordinates are just given in (3.4b) (see fig. 1). Furthermore, in $C$ we have

$$
V^{*}=\frac{1}{2} \sum_{j=1}^{k} \mathcal{N}_{i} \lambda_{i}
$$

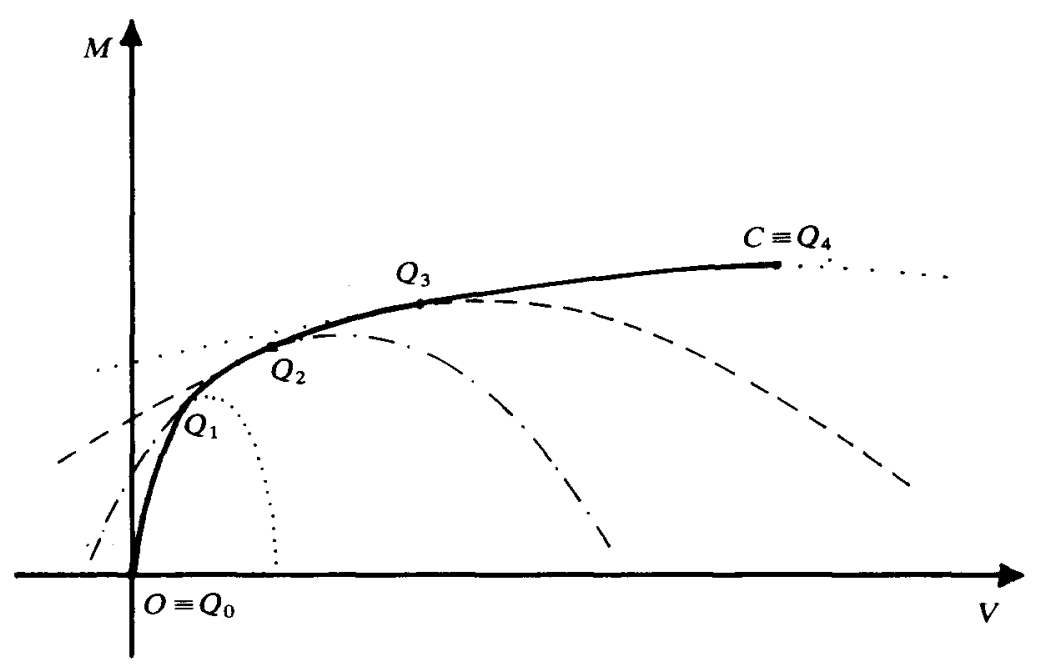

FIGURE 1. Efficient frontier with four risk classes.

To solve the conditional extremum problem, let us set up the Lagrangian function

$$
L(\boldsymbol{x}, \mu)=V(\boldsymbol{x})+\mu[M-M(\boldsymbol{x})] .
$$

From the equations $\partial / \partial x_{j} L(x, \mu)=0$ we obtain

$$
x_{i}=\frac{\lambda_{i}}{2}\left(\frac{1}{\mu}+\eta_{i}^{*}+2\right), \quad(j=1,2, \ldots, k) .
$$

These are the parametric equations of a straight line $O$ which passes through the center $C$ of the interval $D$ and coincides with its "upward" diagonal (i.e., 
the straight line passing through points $\left(\lambda_{1}, \lambda_{2}, \ldots, \lambda_{k}\right)$ and $\left.\left(x_{1}^{*}, x_{2}^{*}, \ldots, x_{k}^{*}\right)\right)$ only if the values of $\eta_{j}^{*}$ are all equal.

By substituting (3.6) in the constraint equation, one has

$$
\frac{1}{\mu}= \pm 2 \sqrt{\sum_{j=1}^{k} \frac{\mathcal{N}_{i} \lambda_{j}}{\eta_{i}^{*}}} .
$$

The negative roots of (3.7) are to be discarded because, as it is easy to verify, they correspond to points with maximum variance. Therefore all the points of the straight line $O$ that are lower than $C$ are to be discarded as inefficient. Then equation (3.7), modified in this manner, leads to the parametric equations of $\mathcal{O}$, $x_{j}=x_{j}(M)$. To obtain the efficient frontier it is sufficient to substitute these expressions of $x_{j}$ into the objective function, thus attaining $V=V(M)$ and therefore, passing to the inverse function, the equation

$$
M=-\frac{V^{2}}{\sum_{j=1}^{k} \frac{\mathcal{N}_{j} \lambda_{j}}{\eta_{j}^{*}}}+\frac{\sum_{j=1}^{k} \mathcal{N}_{i} \lambda_{j}}{\sum_{j=1}^{k} \frac{\mathcal{N}_{j} \lambda_{j}}{\eta_{j}^{*}}} V+M^{*}-\frac{1}{4} \frac{\left(\sum_{i=1}^{k} \mathcal{N}_{j} \lambda_{i}\right)^{2}}{\sum_{j=1}^{k} \frac{\mathcal{N}_{j} \lambda_{j}}{\eta_{j}^{*}}}
$$

It is to be noted that the constant term in (3.8) is nonnegative and vanishes if and only if the $\eta_{i}^{*}$ values are all equal. In fact, by indicating by $A\left(\left\{\eta_{i}^{*}\right\}\right)$ the weighted arithmetic mean of the $\eta_{j}^{*}$, with weighting factors $\mathcal{N}_{j} \lambda_{j} / \sum_{j=1}^{k} \mathcal{N}_{j} \lambda_{j}$, it can be written, keeping in mind (3.4a)

$$
\frac{1}{4} \sum_{j=1}^{k} \mathcal{N}_{i} \lambda_{j}\left[A\left(\left\{\eta_{j}^{*}\right\}\right)-\frac{1}{A\left(\left\{1 / \eta_{i}^{*}\right\}\right)}\right]
$$

and the conclusion is drawn by observing that the quantity between square brackets is the difference between the arithmetic and the harmonic mean.

In order that equation (3.8) represents an efficient frontier it is necessary to bound it to suitable values of $V$. Above all we shall disregard values greater than $V^{*}$, in so far as they provide levels of expected return less than $M^{*}$ (and in fact they are the points lower than $C$, which we have discarded because of the inversion of $V=V(M)$ ). Values of the variance that are decreasing from $V^{*}$ corresponds to points of $\mathcal{O}$ which move upwards away from $C$, until they reach the boundary of $D$. We shall denote by $Q_{k-1}$ the intersection point between $O$ and this boundary. If all the $\eta_{j}^{*}$ were to be equal, the point $Q_{k-1}$ would coincide with the vertex $\left(x_{1}^{*}, x_{2}^{*}, \ldots, x_{k}^{*}\right)$ of $D$, that we shall indicate by $Q_{0}$ and that corresponds to values of $M$ and $V$ equal to zero (empty portfolio). Instead, in the general case, the first class to be excluded will be the one corresponding to the least $\eta_{j}^{*}$.

Let us then consider a permutation $q$ of the subscripts $\{j\}$ such that

$$
\eta_{q 1}^{*} \leqslant \eta_{q 2}^{*} \leqslant \cdots \leqslant \eta_{q k}^{*}
$$


The coordinates of the point $Q_{k-1}$ will then be expressed by

$$
x_{j}\left(Q_{k-1}\right)=\left(1+\frac{\eta_{q 1}^{*}+\eta_{j}^{*}}{2}\right) \lambda_{j}, \quad(j=1,2, \ldots, k)
$$

which provide

$$
\begin{gathered}
V\left(Q_{k-1}\right)=V^{*}-\frac{\eta_{q 1}^{*}}{2} \sum_{j=1}^{k} \frac{\mathcal{N}_{j} \lambda_{j}}{\eta_{j}^{*}}, \\
M\left(Q_{k-1}\right)=M^{*}-\left(\frac{\eta_{q 1}^{*}}{2}\right)^{2} \sum_{j=1}^{k} \frac{\mathcal{N}_{j} \lambda_{j}}{\eta_{j}^{*}} .
\end{gathered}
$$

The value $V\left(Q_{k-1}\right)$ is the minimum possible variance relative to an efficient portfolio made up of $k$ risk classes. For lower values of $V$ we are led in practice to a problem in $k-1$ dimensions, until the increase of the prices charged will not bring to the exclusion of the class $\mathscr{C}_{q 2}$. This will happen in a point $Q_{k-2}$ with coordinates

$$
x_{j}\left(Q_{k-2}\right)= \begin{cases}\left(1+\frac{\eta_{a 2}^{*}+\eta_{i}^{*}}{2}\right) \lambda_{i}, & \text { for } j \neq q_{1} \\ x_{j}^{*}, & \text { for } j=q_{1} .\end{cases}
$$

The efficient portfolios composed of $k-1$ risk classes are represented by the points of the line-segment $Q_{k-1} Q_{k-2}$, laying on the boundary of $D$ and the efficient frontier has the same expression as in (3.8), provided that now we bound it to the values of $V$ contained between $V\left(Q_{k-2}\right)$ and $V\left(Q_{k-1}\right)$ and the sums range over the remaining $k-1$ classes. By continuing to increase the prices, the progressive elimination of all the risk classes will be brought about, until one reaches, in $Q_{0}$, the emptying out of the portfolio.

The complete efficient frontier can be expressed by

$$
\left\{\begin{aligned}
\boldsymbol{M}= & \left(\sum_{j=1}^{k} \frac{\mathcal{N}_{q j} \lambda_{q j}}{\eta_{q i}^{*}}\right)^{-1}\left\{-V^{2}+\left(\sum_{j=1}^{k} \mathcal{N}_{q j} \lambda_{q i}\right) V\right. \\
& \left.+\frac{1}{4}\left[\sum_{j=1}^{k} \frac{\mathcal{N}_{q j} \lambda_{q j}}{\eta_{q j}^{*}} \sum_{j=1}^{k} \mathcal{N}_{q j} \lambda_{q j} \eta_{q j}^{*}-\left(\sum_{j=1}^{k} \mathcal{N}_{q j} \lambda_{q i}\right)^{2}\right]\right\}, \\
& \text { for } V\left(Q_{k-s}\right)<V \leqslant V\left(Q_{k-s+1}\right), \quad(s=1,2, \ldots, k),
\end{aligned}\right.
$$

where we denote $C=Q_{k}$, and the points $Q_{k-s}$ have coordinates

$$
x_{j}\left(Q_{k-s}\right)= \begin{cases}\left(1+\frac{\eta_{q s}^{*}+\eta_{j}^{*}}{2}\right) \lambda_{j}, & \text { for } j \neq q_{1}, q_{2}, \ldots, q_{s-1} \\ x_{j}^{*}, & \text { for } j=q_{1}, q_{2}, \ldots, q_{s-1} .\end{cases}
$$

In the space $\mathbb{R}^{k}$ the efficient portfolios are represented by the points of the broken line $C Q_{k-1} \ldots Q_{1} Q_{0}$. 
In the plane $(V, M)$ the efficient frontier has the shape of a "chain" of arcs of parabola that are joined together in the points $Q_{k-s}$ and which are ever more convex from $C$ to $Q_{0}$.

\subsection{Maximizing the Expected Utility of the Company}

Once the efficient frontier has been determined, the analysis of the decision problem of the company is concluded by choosing the portfolio that represents the best trade-off between mean and variance, that is by maximizing a utility function of the form $u(M, V)$. By introducing a set of indifference curves in the $(V, M)$ space, the optimal portfolio is represented by the tangency point between the efficient frontier and the indifference curve corresponding to the highest possible level of the utility.

If we suppose, for example, that the initial free capital $w$ is large relative to the expected return on the portfolio, i.e., if $w \gg M^{*}$, then it is possible, analogously as was done in sect. (2.4), to approximate the utility function of the company by the quadratic utility function

$$
u(w+z)=z-\frac{1}{2} r_{c}(w) z^{2},
$$

with the related indifference curves

$$
V=-M^{2}+\frac{2}{r_{c}(w)}(M-U),
$$

where $U$ is the level of expected utility corresponding to the curve and $r_{c}(w)$ represents the Arrow-Pratt measure of risk aversion of the insurance company.

However it is to be noted that, with our assumptions, the suitability in using the variance as a measure of the riskiness of the portfolio does not rely on the accuracy of the quadratic approximation of the company's utility function, but on the goodness of the approximation made for the probability of ruin.

\section{ILLUSTRATION OF RESUltS IN THE TWO-ClASSES CASE}

Let us now discuss and illustrate the results obtained in section (3) in the case in which the risk market is made up of only two risk classes $\mathscr{C}_{1}$ and $\mathscr{C}_{2}$, with $\lambda_{1}<\lambda_{2}$. Furthermore, let suppose that $\mathscr{C}_{1}$ is characterized by a level of risk aversion greater ti an $\mathscr{C}_{2}$, i.e., $\eta_{1}^{*}>\eta_{2}^{*}$.

The problem finds a simple geometric representation in the plane $\left(x_{1}, x_{2}\right)$ (see fig. 2). We see that the level lines $M(x)=M$ of the expected return constitute a set of ellipses with center $C$ (the center of rectangle $D$ ), axes parallel to the coordinate axes and size decreasing as $M$ increases. The maximum expected return will then be attained by choosing the premiums $x_{j}=x_{j}(C)$. The level lines $V(x)=V$ of the variance instead form a set of parallel straight lines with slope $-\mathcal{N}_{1} \eta_{2}^{*} / \mathcal{N}_{2} \eta_{1}^{*}$ that come closer to the origin as $V$ increases. 


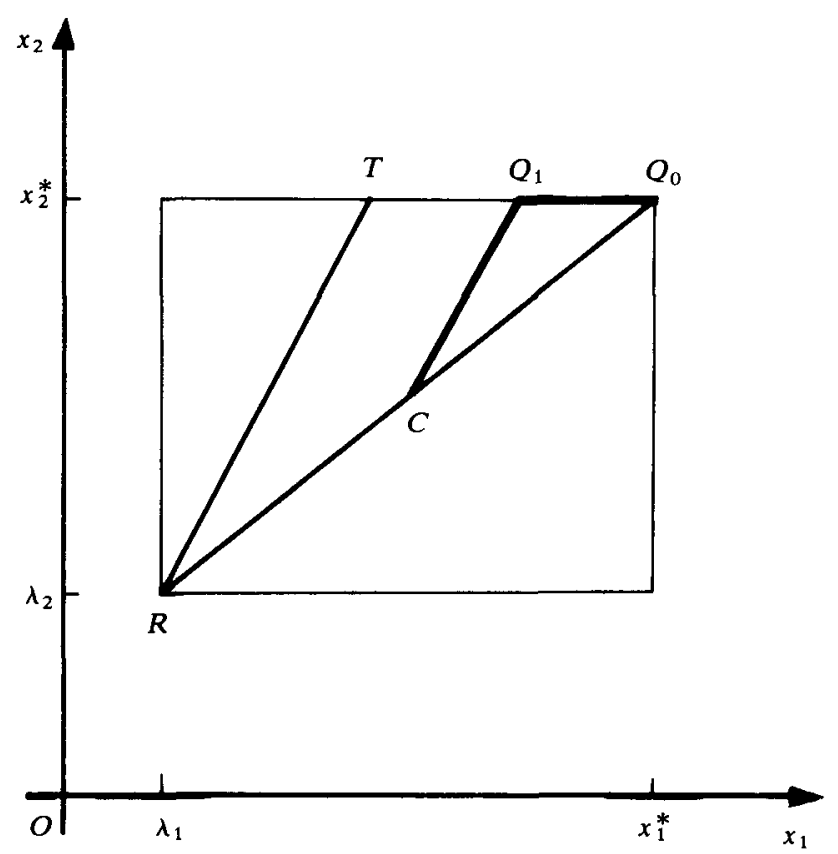

FIGURE 2. $C Q_{1} Q_{0}$ : Efficient prices; $R Q_{0}$ : Market portfolios; $R T$ : Proportional loadings.

The efficient portfolio relative to the choice of a return level $M$ is therefore represented by the tangency point between the ellipse $\boldsymbol{M}(\boldsymbol{x})=\boldsymbol{M}$ and the "highest" possible variance level line. In this manner we obtain the straight line $\mathcal{O}$

$$
x_{2}=\frac{\lambda_{2}}{\lambda_{1}} x_{1}-\frac{1}{2} \lambda_{2}\left(\eta_{1}^{*}-\eta_{2}^{*}\right),
$$

which passes through $C$ and intersects the boundary $x_{2}=x_{2}^{*}$ of $D$ in the point $Q_{1}$, having abscissa $x_{1}\left(Q_{1}\right)=\left[1+\left(\eta_{2}^{*}-\eta_{1}^{*}\right) / 2\right] \lambda_{1}$. Of course, from $O$ are to be discarded, besides the points above $Q_{1}$, even those below $C$, that correspond to inefficient portfolios (maximum $V$ for given $M$ ). The prices indicated by $Q_{1}$ generate the mixed portfolio with minimum variance; in order to achieve lesser values of $V$ it is necessary to operate with only one class, choosing the prices on the line-segment $Q_{1} Q_{0}$.

If the two classes were to have an equal degree of risk aversion, i.e., if $\eta_{1}^{*}=\eta_{2}^{*}=\eta^{*}$, the locus of the efficient solutions would be given by

$$
x_{j}=\lambda_{j}\left(1+\frac{\eta^{*}}{2}+\frac{1}{\mu}\right)
$$

with

$$
0 \leqslant \frac{1}{\mu} \leqslant \frac{\eta^{*}}{2}, \quad(j=1,2)
$$


and the points $Q_{1}$ and $Q_{0}$ would coincide. In this manner a premium-making policy of rather intuitive significance would be confirmed, that is the charging to both the classes of a loading equal to the same percentage $\eta$ of the net premium. In our case, instead, being $\eta_{1}^{*}>\eta_{2}^{*}$, it turns out that the efficient choices consist in overloading the more risk averse class $\mathscr{C}_{1}$ by increasing the percent loading $\eta \lambda_{1}$ by the quantity $\left(\eta_{1}^{*}-\eta_{2}^{*}\right) / 2$.

Another interesting result consists in the fact that a diversification of the portfolio is not always efficient, because if small values of the variance (linesegment $Q_{1} Q_{0}$ ) are desired, then the expected return is maximized by insuring only individuals that are of the more risk averse class.

Finally, let us compare the policy of the efficient prices with that of the prices that determine a natural, or market, portfolio, that is a portfolio that contains both the risk classes in the same proportion with which they are present on the market. By solving the equations

$$
\frac{n_{j}\left(x_{j}\right)}{\sum_{j=1}^{k} n_{j}\left(x_{j}\right)}=\frac{\mathcal{N}_{j}}{\sum_{j=1}^{k} \mathcal{N}_{j}}, \quad(j=1,2),
$$

one easily obtains the parametric equations

$$
\left\{\begin{array}{l}
x_{j}=x_{j}^{*}-d \eta_{j}^{*} \lambda_{j}, \\
0 \leqslant d \leqslant 1, \\
(j=1,2),
\end{array}\right.
$$

that represent the diagonal of $D$ passing through $Q_{0}$. As can be seen, it is a matter of charging to the two classes a loading which is equal to a same fraction $(1-d)$ of the respective maximum percent loading $\eta_{i}^{*}$ and this policy will turn out to be efficient if the classes are characterized by a different degree of risk aversion.

\section{REFERENCES}

AMMETER, H. (1970) Das Solvibilitätsproblem in der Risiko-Lebenversicherung, Mitteilungen der Vereinigung schweizerischer Versicherungsmathematiker 70, 41-56.

Ammeter, H., DePOID, P. and DE FINETTI, B. (1959) L'Étude Mathématique des Assurances Non Viagères dans l'Europe Continentale Occidentale. Astin Bulletin 1, 46-70.

AMSLER, M.-H. (1978) L'equation générale d'équilibre d'un risque collectif. Mitteilungen der Vereinigung schweizerischer Versicherugsmathematiker 78, 221-246.

BühlmanN, H. (1980) An Economic Premium Principle. Astin Bulletin 11, 52-60.

CACCIAfESTA, R. (1970) Sulla Determinazione delle Classi di Rischio. Giornale dell'Istituto Italiano degli Attuari 70, 38-48.

Dubourdieu, J. (1952) Théorie Mathématiques des Assurances. I, Théorie Mathématique du Risque dans les Assurances de Répartition. Gauthier-Villars: Paris.

FERRA, C. DE (1968) Sulla Vantaggiosità delle Operazioni Assicurative, Giornale dell'Istituto Italiano degli Attuari 68, 1-16.

FinetTI, B. DE (1939) La Teoria del Rischio e il Problema della "Rovina dei Giocatori". Giornale dell'Istituto Italiano degli Attuari 39, 41-51. 
Gerber, H. U. (1981) On the Probability of Ruin in an Autoregressive Model. Mitteilungen der Vereinigung schweizerischer Versicherungsmathematiker 81, 213-219.

JONG, P. DE (1981) Insurance Premiums under Demand Constraints. Scandinavian Actuarial Journal 81, 123-125.

Kahane, Y. (1979) The Theory of Insurance Risk Premiums-A Re-examination in the Light of Recent Developments in Capital Market Theory. Astin Bulletin 10, 223-239.

LuNDBERG, F. (1909) Über die Theorie der Rückversicherung. Trans. VI Intern. Congr. Act. 1, 877-948.

Massé, P. (1964) Le Choix des Investissements (Critéres et Méthodes). Dunod: Paris.

PressacCo, F. (1979) Value and Prices in a Reinsurance Market. Astin Bulletin 10, 263-273.

RothsChild, M. and Stiglitz, J. (1976) Equilibrium in Competitive Insurance Markets: an Essay on the Economics of Perfect Information. Quarterly Journal of Economics 90, 629-649.

SEAL, H. L. (1979) Survival Probabilities (The Goal of Risk Theory). Wiley: New York.

SENGUPTA, J. K. (1981) Optimal Decisions under Uncertainty. Lecture Notes in Economics and Mathematical Systems N.193, Springer-Verlag: Berlin.

Volpe di Prignano, E. (1974) Sulla Misurabilità del Premio di Indifferenza nei Rami Elementari. Giornale dell'Istituto Italiano degli Attuari 74, 25-45. 